\section{Transcription prescription for herpes}

\section{By Tracey Baas, Associate Editor}

Marketed drugs used to treat herpes simplex virus and varicella zoster virus share a single mechanism: inhibiting viral DNA polymerase to prevent replication of the viral genome. Now, NIH researchers have found a new way to attack herpes infections-stopping the virus from hijacking the host's transcriptional machinery to produce viral mRNA. The technique involves blocking a host enzyme, lysine-specific demethylase-1, which the virus exploits to kick-start transcription of viral mRNA.

Because $\alpha$-herpesviruses are DNA viruses, they lack their own RNA polymerase and therefore require the use of the host's. To prepare the host's transcriptional machinery for viral gene transcription, $\alpha$-herpesviruses modify methylation levels of host histones. In particular, the virus needs to increase methylation of histone H3 Lys4 (H3K4) and decrease methylation of H3K9.

The mechanism behind the former task is well established (see Figure 1, "LSD1 regulation of viral gene transcription"), but the precise host demethylase the virus uses on $\mathrm{H} 3 \mathrm{~K} 9$ has remained elusive.

A team led by Thomas Kristie, chief of the molecular genetics section group at the National Institute of Allergy and Infectious Diseases (NIAID), pinpointed lysine-specific demethylase-1 (LSD1) as the mystery demethylase. ${ }^{1}$ In vitro and ex vivo studies showed that knocking down or inhibiting LSD1 decreased levels of $\alpha$-herpesvirus mRNA and proteins.

The work was published in Nature Medicine.

Kristie said he and his colleagues decided to focus on LSD1 as a potentially targetable player in viral infection because "at the time in which our study began, LSD1 was one of the few characterized histone demethylases."

The group tested whether a known class of small molecule inhibitors of LSD1 could curb viral infection. The class they chose inhibits monoamine oxidases (MAOs), a target that has high sequence homology to LSD1. Previous work by other groups has shown that MAO inhibitors (MAOIs), which are on the market for a variety of neurological conditions, also hit LSD1.,3

"For years it's been remarked that patients on MAO inhibitors are notably unlikely to come down with viral illnesses," said Alexander Bodkin, director of the clinical psychopharmacology research program at McLean Hospital. He is a co-developer of Emsam selegi- line, a transdermal MAOI patch that is marketed by Mylan Inc. to treat major depressive disorder.

Kristie's team treated herpes simplex virus (HSV)- or varicella zoster virus (VZV)-infected cells with the MAOI tranylcypromine. The researchers saw dose-dependent decreases in viral immediateearly mRNA and proteins. The drug also lowered the amount of HSV by nearly three orders of magnitude compared with vehicle.

Those studies focused on the lytic phase of the virus, during which viral replication, assembly and spread take place. $\alpha$-Herpesviruses can also enter a latent phase, during which they retreat to neural ganglia in the nervous system and can be reactivated into the lytic phase when conditions are right.

In this setting, tranylcypromine also significantly reduced reactivation of HSV in murine trigeminal ganglion explants compared with vehicle $(p<0.005)$. Viral reactivation occurred in only $7 \%$ of tranylcypromine-treated ganglia compared with $75 \%$ of ganglia treated with acyclovir, a generic inhibitor of viral DNA polymerase.

"The work shows that the MAO inhibitors target the expression of HSV genes that are required both during the reactivation phase and also during normal lytic viral replication via a mechanism completely different than that of acyclovir," said William Ruyechan, professor in the Department of Microbiology and Immunology at the State Uni-

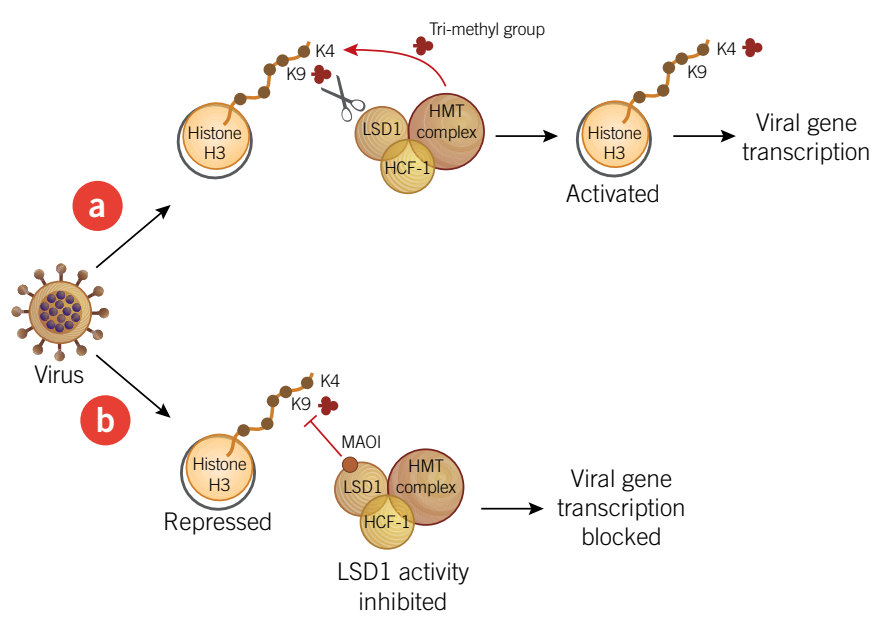

Figure 1. LSD1 regulation of viral gene transcription. To prepare a host for viral gene transcription, $\alpha$-herpesviruses need to increase methylation of histone H3 Lys4 (H3K4) and decrease methylation of $\mathrm{H} 3 \mathrm{~K} 9$. To increase methylation, the virus recruits host cell factor-1 (HCF-1) and a histone methyltransferase (HMT) complex [a]. Researchers reported in Nature Medicine that to decrease methylation, a demethylase called lysine-specific demethylase-1 (LSD1) is required. LSD1 interacts with the HCF-1 component of the histone methyltransferase complex.

The researchers also showed that blocking LSD1 activity using monoamine oxidase inhibitors (MAOls), which are known to target LSD1, led to inhibition of viral gene transcription [b]. 


\section{Box 1. Crystallized thoughts on LSD1.}

NIH researchers have shown that lysine-specific demethylase-1 could be a good target to treat herpes infections. ${ }^{1}$ Separately, Frontier Pharma Inc. and Japanese academics have designed a new class of selective lysine-specific demethylase-1 antagonists. ${ }^{4}$ Although the Japanese researchers first plan to test their compounds in cancer, in which aberrant DNA methylation patterns are a disease hallmark, the $\mathrm{NIH}$ findings have prompted them to consider studies of the antagonists in infections.

Naoki Miyata, professor of organic and medicinal chemistry at Nagoya City University, and colleagues at the Nagahama Institute of Bio-Science and Technology and Frontier Pharma, analyzed existing crystal structures of lysine-specific demethylase-1 (LSD1) bound to different molecules: tranylcypromine or $N$-propargyl lysine-containing H3 peptides. Those two molecules inhibit LSD1, but both have problems that have prevented their development as therapeutics.

For example, tranylcypromine also targets monoamine oxidases (MAOs) and thus could have side effects, whereas the $N$-propargyl lysine-containing $\mathrm{H} 3$ peptides have difficulty permeating membranes.

Based on the crystal data, the researchers decided the best way to proceed would be to combine the tranylcypromine backbone and the lysine group from the peptides.

The resulting small molecule tranylcypromine-lysine analogs showed LSD1 selectivity that was 400-11,000 times greater than that of tranylcypromine. In human cancer cell lines, the compounds inhibited cell growth at concentrations of 6-67 $\mu \mathrm{M}$.

The work was published in the Journal of the American Chemical Society.

Based on the NIH findings published in Nature Medicine,${ }^{1}$ Miyata said there is a good chance that his team's compounds also might work as antiHSV agents. He said he is interested in seeing if the compounds are active in experiments similar to those reported in Nature Medicine.

Miyata also told SciBX that his group is "very interested in examining the antiviral activity of our LSD1-selective inhibitors against viruses other than HSV." Indeed, the group at Nagoya City University first plans to study the antiviral activity of its LSD1-selective inhibitors against HIV. Meanwhile, Frontier Pharma will take the lead in preclinical studies of the compounds to treat cancer.

Frontier Pharma is housed at the Nagahama Bio Incubation Center. Nagoya City University has filed for a patent covering the findings reported in JACS. Miyata said the IP is available for licensing. $-T B$ versity of New York at Buffalo. "This paper identifies a new target: viral transcription as opposed to the classic DNA replication target."

David Knipe, professor of microbiology and molecular genetics and chair of the virology program at Harvard Medical School, said the study "provides important new basic science on the role of chromatin in herpes simplex virus replication and proof of concept that pharmacological control of histone-modifying enzymes can block HSV lytic replication and reactivation from latency."

\section{MAO dynasty}

Kristie's next step is in vivo experiments. "The next stages in the project would be to assess the impact of MAO inhibitor treatment in a mouse model system of HSV infection to determine the ability of these drugs to prevent HSV pathogenesis and prevent reactivation of latent virus," he told SciBX.

According to Richard Whitley, the mouse is a good model in which to start evaluating latency and HSV-mediated CNS disease. He and other researchers at The University of Alabama at Birmingham will be collaborating with Kristie to test LSD1 inhibition in mouse models of HSV latency and HSV-mediated CNS disease and in a Guinea pig model of genital herpes reactivation.

Whitley is a professor in the Department of Medicine at University of Alabama and was the first clinician to use acyclovir in humans for neonatal HSV and herpes simplex encephalitis.
He said that acyclovir is good for uncomplicated herpes infections; however, for difficult cases such as neonatal HSV and herpes simplex encephalitis, something better is needed. "Because MAO inhibitors are used in the clinic to treat disorders of the brain, we already know that they likely transverse the blood brain barrier," he added.

In addition to testing MAOIs in vivo, Kristie said the development of specific LSD1 inhibitors could yield even better therapeutics for various herpes infections.

Dewey McCafferty, professor of chemistry and biochemistry at the Levine Science Research Center at Duke University, is doing just that with a series of tranylcypromine analogs. "Our work is centered on establishing LSD1-selective inhibitors and confirming their biological activity and molecular mechanism of action," he said.

McCafferty and colleagues were the first to show that tranylcypromine inhibited histone demethylation and was the most potent of the MAOIs for human LSD1 but had limited selectivity for MAOs over LSD1. ${ }^{2,3}$

"Transforming compounds of this structural class into specific LSD1 inhibitors for anti-HSV therapy certainly starts from a position of distinct advantage" versus starting with an entirely new inhibitor structural class for which no pharmacological and toxicological information is available, he said.

McCafferty told SciBX that it is not yet clear whether tranylcypro- 
mine analogs will cross-react with some MAOs, which could lead to adverse events. For example, some nonselective MAOIs can interfere with MAO-dependent metabolism of tyramine from tyramine-containing foods such as aged cheese and cured meat, which leads to tyramine-associated toxicity. Nevertheless, he said, "I remain confident and optimistic that selective inhibitors may be discovered for this class of enzymes.” (See Box 1, “Crystallized thoughts on LSD1.”)

The NIH has filed patent applications covering methods of preventing or treating viral infection or reactivation from latency in a host using inhibitors of the LSD1 protein. The IP is available for licensing through the NIAID Office of Technology Development.

Baas, T. SciBX 2(46); doi:10.1038/scibx.2009.1683

Published online Dec. 3, 2009

\section{REFERENCES}

1. Liang, Y. et al. Nat. Med.; published online Oct. 25, 2009; doi:10.1038/nm.2051

Contact: Thomas M. Kristie, National Institutes of Health, Bethesda, Md. e-mail: thomas_kristie@nih.gov

2. Schmidt, D. \& McCafferty, D. Biochemistry 46, 4408-4416 (2007)
3. Lee, M. et al. Chem. Biol. 13, 563-567 (2006)

4. Ueda, R. et al. J. Am. Chem. Soc.; published online Nov. 16, 2009; doi:10.1021/ja907055q

Contact: Naoki Miyata, Nagoya City University, Aichi, Japan e-mail: miyata-n@phar.nagoya-cu.ac.jp

Contact: Takayoshi Suzuki, same affiliation as above e-mail: suzuki@phar.nagoya-cu.ac.jp

Contact: Tamio Mizukami, Nagahama Institute of Bio-Science and Technology, Shiga, Japan

e-mail: mizukami@nagahama-i-bio.ac.jp

\section{COMPANIES AND INSTITUTIONS MENTIONED}

Duke University, Durham, N.C.

Frontier Pharma Inc., Shiga, Japan

Harvard Medical School, Boston, Mass.

McLean Hospital, Belmont, Mass.

Mylan Inc. (NASDAQ:MYL), Canonsburg, Pa.

Nagahama Institute of Bio-Science and Technology, Shiga, Japan

Nagoya City University, Aichi, Japan

National Institute of Allergy and Infectious Diseases, Bethesda, Md.

National Institutes of Health, Bethesda, Md.

The University of Alabama at Birmingham, Birmingham, Ala.

State University of New York at Buffalo, Buffalo, N.Y. 